\title{
Perfil sociodemográfico y de intereses profesionales de los estudiantes de licenciatura en enfermería
}

\section{Socio-demographic profile and professional interests of students of Bachelor's degree in nursing}

María de Jesús de Valle Alonso• Imelda Elena Hernández López•

Perla Martínez Aguilera.• Sonia Yolanda Barrón Cabrera.•

Martha López Hernández• Martha Lilia Zúñiga Vargas.•

\section{Resumen}

Introducción: Aunque en los servicios de salud a nivel mundial existe deficiencia de personal de enfermería, en América Latina el índice de deserción en las escuelas de enfermería es elevado; esta problemática recae en trabajadores en ejercicio quienes deben brindar cuidado enfermero en situaciones de cansancio y estrés. La problemática lleva a pensar que no existen características adecuadas en los estudiantes que ingresan para cursar favorablemente la carrera. Objetivo: Determinar las características sociodemográficas, económicas, familiares, de antecedentes escolares e intereses profesionales de estudiantes que ingresan a estudiar la carrera de Enfermería. Suponemos una relación entre deserción e intereses u objetivos que guiaron a alumnos en la selección de carrera, sus características socio-económicas-familiares y del plantel que provienen. Metodología: Estudio transversal y descriptivo, se consideró el 100\% de las cédulas de tutoría aplicadas a estudiantes de Enfermería que ingresaron entre 2001 y 2006. Los datos sociodemográficos, económicos y familiares se obtuvieron por medio de preguntas abiertas o cerradas, para clasificar nivel socioeconómico se utilizó la propuesta de Sigmarket, los intereses profesionales se evaluaron a partir de una escala tipo likert. Resultados: El perfil obtenido mostró una población joven, predominantemente solteros, que no realizan trabajo remunerado, dependen económicamente de sus padres, de condiciones socioeconómicas precarias, sus intereses están orientados hacia la obtención de conocimientos y aspectos de carácter social Conclusiones: Fue importante identificar una tendencia, aunque no significativa estadísticamente, de permanencia en mayor medida de los alumnos con condiciones sociales más precarias, y aquellos cuyos intereses de orden social se ubican en el nivel más alto.

- Maestría en Educación Superior, Profesor de tiempo completo, Facultad de Enfermería Nuevo Laredo (UAT).

- Maestría en Ciencias de la Enfermería, Profesor de tiempo completo, Facultad de Enfermería Nuevo Laredo (UAT).

...Licenciada en Enfermería, Profesor de tiempo completo, Facultad de Enfermería Nuevo Laredo (UAT).

Correspondencia: mjdevalle@uat.edu.mx

FeCha DE ReCIBIDO: 10 DE JULIO 2011

Fecha de Enviado: 03 De Septiembre 2011

Fecha de Aceptado: 5 De noviembre 2011 


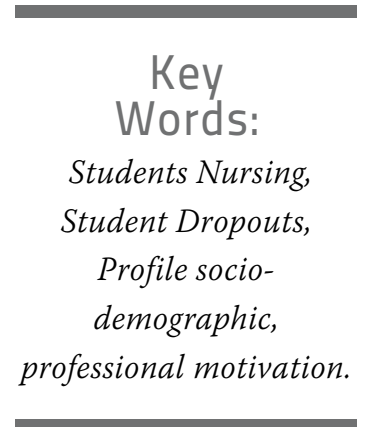

ABstract

Introduction: Although it exists a deficiency in nursing staff at a global level in health services, in Latin America the rate of dropout in nursing schools is high; this problem lies over workers who must provide nurse care in situations of fatigue and stress. The problem leads us to think there are no appropriate characteristics for students to successful fulfill their career. Objective: to determine the socio demographic, economic and family characteristics, school background and professional interests of students entering nursing. We assume an existing relationship between dropout, the interests or goals that guided students in the selection of the career in nursing, the socio-economic-family characteristics and the institution of origin. Methodology: cross-sectional and descriptive study that considered the 100\% of admission interviews made to incoming nursing students from 2001 to 2006. The socio-demographic, economical and familiar data were obtained from closed and open-ended questions; the Sigmarket proposal was used for the classification of the socioeconomic level, the professional interests were evaluate from a likert scale. Results: the obtained profile showed a young population, mainly single, that doesn't have a paid job and depends from their parents economically, from a low socio-economic conditions, their interests are focus towards the acquisition of knowledge and aspects of social nature. Conclusions: it was important to identify a mayor tendency, although not statistically significant, of continuing the career from part of the students with lower social conditions and those whose social interests are located in the highest level.

\section{INTRODUCCIÓN}

Aunque en los servicios de salud a nivel mundial existe deficiencia de personal de enfermería, en América Latina el índice de deserción en las escuelas de enfermería es elevado. Un ejemplo de ello es que en cuatro instituciones universitarias de Perú, formadoras de enfermeras, hasta un $86 \%$ de los alumnos desertaron durante los primeros semestres de escolaridad; específicamente durante el primer año de la carrera. ${ }^{1}$

En México, Nigenda y cols. ${ }^{2}$ estimaron la tasa de desperdicio escolar (abandono definitivo de los estudios) de personas que habían ingresado a estudios de enfermería a nivel de licenciatura, a partir de datos recopilados por la ANUIES durante el periodo de 1978-2002 que incluyó a 22 generaciones. Las tasas de abandono, aunque con mucha variación, muestran tendencia a la disminución, en 1987 fue de 555 por cada mil, en cambio para la generación que concluyó en el año 2002 se ubicó en 252 por cada mil, no obstante de acuerdo con este último dato uno de cada cuatro alumnos desertan.

Rodríguez y Hernández ${ }^{3}$ utilizan también datos de la ANUIES para analizar la deserción en general en las universidades mexicanas durante el periodo 1999-2003, identifican un mayor porcentaje en las universidades públicas en comparación a las privadas, 36 vs $29 \%$ respectivamente, aunque el porcentaje promedio está en alrededor del $25 \%$, conforme a estos datos los correspondientes a enfermería proporcionados por Nigenda están dentro del rango general de todas las carreras, que igualmente es alto.

Pudiera suponerse que las universidades de la frontera norte del país no enfrentan problemas de deserción de estudiantes de enfermería, debido a factores cómo el incremento de fuentes de trabajo para ellos, además de la doble titulación que ha surgido por parte de instituciones de educación superior nacionales y de Norteamérica, así como la contratación de egresados en instituciones de salud en Estados Unidos. No obstante, el escenario observado muestra un $52.6 \%$ de deserción y un $47.4 \%$ de eficiencia terminal en la Institución de Educación Superior (IES) sede del presente estudio. Es decir, poco más de la mitad que ingresa a la carrera de enfermería la abandona; y en esos mismos términos los que 
concluyen la carrera de Licenciado en Enfermería (LE), no se titulan. Además, se ha observado que cada vez es más frecuente que el alumno manifieste que no le gusta asistir a la práctica clínica; habiendo un gran ausentismo en todos los contextos y niveles de salud. Las repercusiones de la falta de profesionales de enfermería en las instituciones de salud públicas y privadas, propicia que no se cubra al personal de descanso o en periodos vacacionales, la resolución de esta problemática recae en los trabajadores en ejercicio quienes deben brindar cuidado enfermero en situaciones de cansancio extremo y estrés.

La problemática descrita, donde se aprecia un porcentaje de deserción considerablemente más elevado que lo reportado a nivel nacional, lleva a pensar que no existen características adecuadas en los estudiantes que ingresan a la IES para cursar favorablemente la carrera de enfermería; entre ellas, intereses u objetivos no afines a esta profesión, que sus recursos son insuficientes para el tiempo y los gastos que la preparación de enfermería demanda; o que los alumnos carecen de apoyo familiar para continuar exitosamente su formación. De tal forma que se plantea la siguiente pregunta: ¿Cuáles son las características sociodemográficas, económicas, familiares, de antecedentes escolares e intereses profesionales, de los estudiantes que ingresan a la Licenciatura en Enfermería?

En la literatura, debido al impacto en la trayectoria de vida de los individuos, el análisis de los factores y procesos vinculados con el ingreso al nivel superior, ha sido un tema motivo de estudio desde principios del siglo pasado, consecuentemen- te se han generado diversas teorías y perspectivas relacionadas con el proceso de elección de carrera, tal vez el ámbito más prolífico es el psicológico, éste aunque pone énfasis en componentes relativos a la personalidad no desconoce el contexto histórico, y puede distinguirse un grupo de teóricos que ven la elección como un medio de satisfacer las necesidades internas. ${ }^{4}$

En el área de enfermería, por las repercusiones que tiene el déficit de este personal en los servicios, el tema formó parte desde 1971 de un estudio internacional auspiciado por la OPS en que se propuso revisar en América Latina, los factores relacionados con la decisión de estudiar enfermería. ${ }^{5}$ Las autoras reconocen que uno de los modelos más utilizados es el de Ginzberg quien sustenta que en la elección de una ocupación se comprometen las necesidades primitivas (deseos) y las exigencias y restricciones (posibilidades), sin embargo tanto este modelo como otros no consideran, de acuerdo a las autoras, los factores estructurales que limitan las alternativas entre las cuales el individuo puede elegir de acuerdo a sus intereses, más aún, sostienen, "los deseos" de los individuos están, en gran medida, moldeados por la posición que ocupa el grupo a que pertenecen. ${ }^{5}$ Este estudio mostró dos aspectos aún vigentes, la influencia de la determinación de género y el peso del prestigio vía el ingreso otorgado a las profesiones universitarias para realizar la selección de carrera. El porcentaje de estudiantes que siguieron un "camino" directo para llegar a estudiar enfermería, es decir, no pensaron estudiar otra carrera y una vez tomada la decisión, tampoco tuvie- ron dudas, fue de $27 \%$ esto es relativamente pequeño.

En cuanto a la deserción, Tinto (citado por Romo y Fresán) identifica cinco teorías que explican éste fenómeno en los estudios realizados en Estados Unidos, atribuyéndolo al contexto: social y ambiental, así como al psicológico, organizacional, además del económico e interaccional. ${ }^{6}$ De acuerdo a este autor, la teoría social y ambiental considera que el sexo, la raza y el estatus social personal son aspectos que colaboran en la predicción del éxito escolar, ya que éstos definen el reconocimiento social general que ocupan los sujetos y las instituciones.

En el área psicológica, se contemplan características personales como la motivación, disposición, capacidad, habilidad y personalidad; y su impacto en el comportamiento universitario. Se asume que estos atributos determinan soluciones distintas a circunstancias formativas similares. La teoría económica admite que los estudiantes valoran los bienes que obtendrán en función de determinado grado que logren en la universidad, con los costos que implica transitar como estudiante universitario. ${ }^{6}$

La teoría organizacional se refiere a la implicación del ámbito educativo ya que la dimensión y la complejidad institucional, el contexto, los estímulos y recursos disponibles de las Instituciones de Educación Superior (IES), trasciende en la socialización de los estudiantes; y por ende, en la permanencia durante la carrera. ${ }^{6}$

Mientras que la interaccional, sustenta que la dinámica que exista entre individuos y su ambiente repercutirán en la conducta escolar del estudiante. ${ }^{6}$ 
Conforme a lo anterior, se puede apreciar que las primeras tres teorías reflejan la relación entre las características o perfil del alumno y el éxito estudiantil. En cambio la teoría organizacional e interaccional resaltan la influencia del contexto institucional y la socialización en la conducta estudiantil.

Estos antecedentes muestran la diversidad de dimensiones y factores que pueden considerarse para investigar tanto la elección de carrera como la deserción, y la estrecha vinculación entre estos dos aspectos. En el ámbito de la enfermería en el país, estos problemas han orientado también diferentes estudios.

En Guanajuato, al analizar los rasgos de personalidad en estudiantes de recién ingreso a la Licenciatura en Enfermería, se identifican aspectos positivos que pudieran favorecer la continuidad en los estudios como: necesidad de causar buena impresión social, sensibles con control de impulsos, adaptables y con flexibilidad ante los requerimientos y necesidad de éxito intelectual. En cuanto a los intereses profesionales, la orientación se inclinó hacia lo social y biológico (que es lo deseable en alumnos de esta área). Mientras que el menor interés fue en lo mecánico y matemático. ${ }^{7}$

El conjunto de características sociodemográficas y familiares de estudiantes de enfermería a nivel técnico en Guadalajara, muestra resultados asociados a jóvenes de nivel socioeconómico bajo, dedicados casi en forma exclusiva al estudio, ya que son solteros y sin hijos, dependientes aún de sus padres y una mínima proporción trabaja. Entre los aspectos relevantes que pudieran contribuir al éxito en su formación es que estudiaron enfer- mería por vocación aunque más de la mitad no recibieron orientación durante su proceso de selección de carrera. Además el vivir bajo el ámbito familiar y contar con una madre con mayor posibilidad de brindar su cuidado y orientación, son, de la misma manera aspectos propicios para la permanencia escolar porque les permite a los estudiantes dedicación en sus estudios. ${ }^{8}$

Otra investigación realizada también con alumnos de enfermería consideró necesario saber, si coincidían las expectativas que tenían al ingreso del rol de enfermería, con sus percepciones en niveles superiores de formación, además de conocer si el rol percibido es congruente con los propósitos de la carrera; al ingreso el $82 \%$ percibe a enfermería como coordinadora de trabajo en equipo; sólo $18 \%$ la identificó como cuidadora; no obstante esta percepción se modifica y el rol en semestres posteriores sí reconoce que la enfermera $(\mathrm{o})$ tiene como responsabilidad el cuidado de individuos/familias, mientras que sólo $2.5 \%$ la ve como ayudante o colaboradora del médico. El que los estudiantes al ingreso, perciban que la enfermera debe integrarse como líder de un equipo de trabajo, es una percepción muy buena para un camino más lejano, sin embargo pocos estudiantes distinguieron al inicio de sus estudios, el cuidado como la esencia de enfermería; $y$ en ese caso, pueden advertir al ir a practicar a las instituciones de salud, un alto grado de desapego a sus propósitos, lo que limitará el término exitoso de sus estudios. ${ }^{9}$

La investigación de Ríos, Olivo y Moreno en la Universidad de Nayarit, en una población de estudiantes de ciencias de la salud, in- cluida enfermería, documenta que en esta población estudiantil se observan las mismas tendencias que a nivel nacional respecto a edad y género, así como otras características mencionadas previamente, en mayor medida solteros, sin hijos, dependientes de los padres, de nivel socioeconómico bajo, dedicados básicamente a sus estudios pues sólo el $17 \%$ trabajan..$^{10} \mathrm{La}$ dependencia de sus padres, y las circunstancias socioeconómicas restringidas, puede en algunos casos limitar pero en otros constituirse en aliciente; este segundo sentido enmarcado en la teoría económi$\mathrm{Ca}^{11}$ plantearía que el contraste entre inversión-retribución motiva a los alumnos para un mayor desempeño escolar y a que culminen con éxito su preparación y desarrollo profesional.

A partir de estas investigaciones es posible apreciar el énfasis otorgado a las características personales (tanto demográficas como psicológicas) y socio-económicasfamiliares en la elección de carrera y en la deserción; en el presente estudio se pretende considerar además factores correspondientes a las instituciones educativas de las que provienen los estudiantes. Así, los objetivos de este estudio fueron: determinar las características socio-demográficas, económicas, familiares, de antecedentes escolares e intereses profesionales de los estudiantes que ingresan a la carrera de LE.

Suponemos una relación entre la deserción y los intereses u objetivos que guiaron a los alumnos en la selección de carrera, sus características socio-económicasfamiliares y las del plantel del que provienen. 


\section{Metodología}

Estudio transversal y descriptivo, se consideró el 100\% (158) de las cédulas de tutoría, aplicadas por los maestros tutores a los estudiantes de LE de nuevo ingreso; en el período comprendido del 2002-1 al 2006-1; se estableció como criterio de inclusión las cédulas con llenado íntegro y contestadas de acuerdo a las instrucciones.

El instrumento utilizado, fue creado en forma colegiada por académicos universitarios del estado, dirigentes del Programa Institucional de Tutorías de la Universidad Autónoma de Tamaulipas. Está integrado en forma semiestructurada por 83 ítems, que para los fines de este estudio agrupamos en los siguientes apartados:

Características sociodemográficas.edad, sexo, estado civil, lugar de procedencia y carrera elegida.

Características económicas.-consistió en definir la situación económica del entorno familiar de los alumnos, como medios para satisfacer las necesidades escolares. Se recurrieron a nueve preguntas cerradas orientadas al ingreso familiar, personas dependientes del aporte económico, propiedad de la vivienda, servicios con los que cuenta, número de cuartos, cantidad de personas que la habitan, auto en la familia, servicios culturales del barrio y recursos escolares en su hogar. Para asignar el nivel económico se utilizó la clasificación de Sigmarket, ${ }^{12}$ especialmente el nivel bajo con sus tres clasificaciones: D+, Dm y D- en donde $\mathrm{D}+$ va de un rango de $\$ 12,791.00$ a $\$ 17,902.00$ con un promedio mensual de \$15,196.00; el Dm de $\$ 7,995.00$ a $\$ 12,790.00$ de ingreso máximo y un promedio mensual de $\$ 10,392.00$. El D- corresponde a un rango de $\$ 4,797.00$ a $\$ 7,994.00$ con promedio de $\$ 6,395.00$. Mientras que el nivel E, clasificado como popular con un mínimo de $\$ 1,199.00$ a $\$ 4,796.00$ con un promedio mensual de $\$ 2,998.00$.

Características familiares. - En lo familiar nos referimos a aspectos educativos y laborales de los padres, la integración familiar y el apoyo económico al estudiante, constó de tres ítems cerrados de opción múltiple y una pregunta abierta, refiriéndose a verificar: con quien vive el estudiante, la ocupación y escolaridad de los padres, y el principal apoyo económico del alumno.

Antecedentes escolares.-particularidades educativas que precedían al nivel universitario en el que se insertaba el estudiante en cuestión; se consideró el promedio, tipo de bachillerato y de escuela de dónde provenía el alumno, sistema de estudios, duración del plan escolar, estudios interrumpidos, además si el alumno trabajaba.

Intereses profesionales.- referidos a la búsqueda de las aspiraciones que señalaron los alumnos al ingreso de la carrera de LE; se evaluaron por medio de una escala tipo Likert constituida por 17 preguntas y un formato de respuesta de cinco puntos que va de 1 = totalmente en desacuerdo, a $5=$ totalmente de acuerdo. Las preguntas de la uno a la cinco se refieren a los intereses del alumno por obtener conocimientos, ejemplo: adquirir conocimientos necesarios para ejercer la profesión; este rubro tiene una puntuación mínima de 5 y máxima de 25. En cuanto a los ítems del seis al trece, aluden a afinidades de orden social, ejemplo: el título universitario es importante para sentirse alguien en la vida; en donde la puntuación fue de $8-40$. En lo referente a los reactivos del catorce al diecisiete se relacionaron al área de desarrollo humano, ejemplo: la universidad es un lugar para desarrollarse como persona, aquí el puntaje fue de 4 a 20. Las puntuaciones en cada rubro se dividieron en tres rangos y de acuerdo a estas se clasificaron en: nivel bajo, medio y alto; en donde a una mayor puntuación corresponde mayor nivel por determinados intereses.

La validez interna del instrumentó se fundamentó en las variables medidas, los atributos subyacentes y la construcción colegiada por expertos, en el Estado. La confiabilidad, se verificó mediante el Alpha de Cronbach el cual fue aceptable (.84). Los datos se capturaron y analizaron a través del programa Statistical Package for the Social Sciences (SPSS) versión 16.

Para la realización del estudio se pidió la aprobación de la dirección de la Facultad sede y del departamento de tutorías para utilizar las cédulas, asimismo se solicitó el consentimiento al Comité de Ética y de Investigación, fundamentándonos en lo referido en el Reglamento de la Ley General de Salud en Materia de Investigación para la Salud (ss) considerando el Titulo Segundo, Capítulo I, Articulo 13; el cual se refiere a la dignidad. $\mathrm{Al}$ artículo 16, que establece la protección a la privacidad de los participantes por lo que la información recabada por el instrumento fue reservada. Esta investigación de acuerdo al Artículo 17, Fracción II perteneció a un estudio de riesgo mínimo, ya 
que sólo se utilizaron los instrumentos auto aplicado para recolectar los datos.

\section{Resultados}

El conjunto de características que conforman el perfil de los alumnos (Tabla 1) nos muestra una población joven (la media de edad fue de 20 años $(D E=4.3)$; con predominio del género femenino (73\%) como es característico de la carrera; en su mayoría son estudiantes solteros (89.2\%) y dedicados sólo a estudiar, ya que únicamente el 21.5\% trabaja.

Respecto a antecedentes escolares (no se incluye en tabla), dos de cada tres provienen de escuelas públicas $(68 \%)$ y uno de cada cinco obtuvo entre 8.1 y 8.5 de promedio de calificación.

Por otra parte las características familiares y socioeconómicas (Tabla 2) son acordes a jóvenes de esta edad pues casi ocho de cada diez viven con sus padres y dependen económicamente de ellos (ya sea de ambos o de uno de ellos), los padres estudiaron primaria y casi todos son empleados, en poco más de la mitad de estos hogares el ingreso familiar es muy precario, de acuerdo a la clasificación socioeconómica de Sigmarket el total de hogares de estos estudiantes corresponde a los niveles bajos (Dm y D-) y, más de la mitad a sectores populares donde su ingreso es apenas de entre 3 a 5 mil pesos (54.4\%), por el número de dependientes también en su mayoría se trata de familias pequeñas ya que en la mitad de los hogares el número de dependientes está entre 2 y 3 personas y una cuarta parte en 4 ó 5 personas. En cuanto a la tenencia de la vivienda tres de cada cuatro familias tienen casa propia.

\section{Tabla 1. Características Individuales*}

$\begin{array}{lll}\text { Edad } & \text { Promedio } & 20 \text { años } \\ \text { Género } & & \% \\ & \text { Femenino } & 73.0 \\ \text { Masculino } & 27.0 \\ \text { Estado Civil } & \text { Solteros } & 89.2 \\ & \text { Casados } & 8.2 \\ & \text { Otro } & 3.0 \\ \text { Trabajo remunerado } & \text { SI } & 21.5 \\ & \text { NO } & 78.5\end{array}$

"Porcentajes calculados respecto a un total de 158 cédulas

Tabla 2. Contexto familiar y socioeconómico*

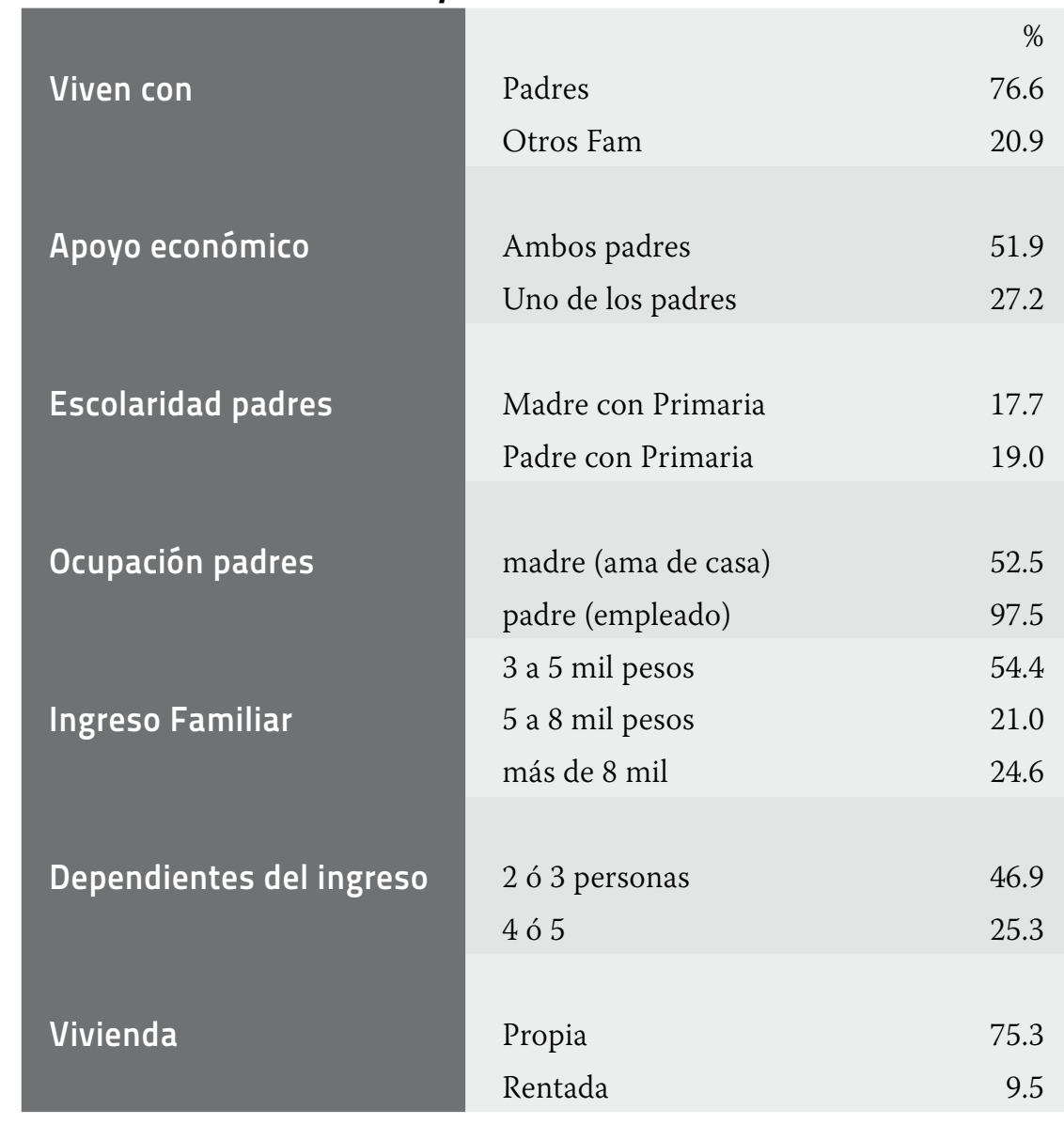

*Porcentajes calculados respecto a un total de 158 cédulas, se muestran los mayores porcentajes 
Con relación a los intereses con los que ingresa el estudiante a la universidad, el $100 \%$ se encontró altamente orientado hacia la obtención de conocimientos, el $74 \%$, refirió medianamente interés por objetivos de orden social y un $33 \%$ se orientó altamente hacia objetivos propios para el desarrollo humano. Esto expresa resultados favorables, ya que es de esperarse que los alumnos vinculen su participación escolar con la obtención de conocimientos y que ello les posibilite además para una mejor comprensión e integración con su entorno social.

Respecto a la deserción y eficiencia terminal, del total (158) de alumnos incluidos en el periodo comprendido del 2002-1 al 2006-1, la eficiencia terminal fue de $55.7 \%$ (88) siendo $76 \%$ mujeres y el $24 \%$ hombres. El 40.9\% (36/88) buscaron la titulación por el Examen General para el Egreso de la Licenciatura (EGEL) de estos $82.3 \%$ fueron mujeres y $22.2 \%$ hombres. Desertó el 21.5\% (34), de estos $61.8 \%$ son mujeres, y $38.2 \%$ varones. Conforme a estos últimos porcentajes pareciera que desertan en mayor medida los varones, sin embargo al aplicar una prueba estadística de chi cuadrada no se encontró la significancia estadística que muestre asociación entre género y la deserción. (Gráfica 1)
Además, conforme a los supuestos planteados se evaluó la relación entre permanencia escolar y nivel socioeconómico con base en el ingreso familiar, la permanencia escolar según el trabajo remunerado y, la permanencia escolar según intereses de orden social. (Gráfica 1) Por los valores relativos obtenidos pareciera que los alumnos cuyo ingreso familiar es precario permanecen en mayor medida que los que tienen mejores ingresos, $y$ aquellos alumnos cuyos intereses de orden social se ubican en el nivel más alto también desertan menos, sin embargo las pruebas de chi cuadrada no alcanzaron significancia estadística.

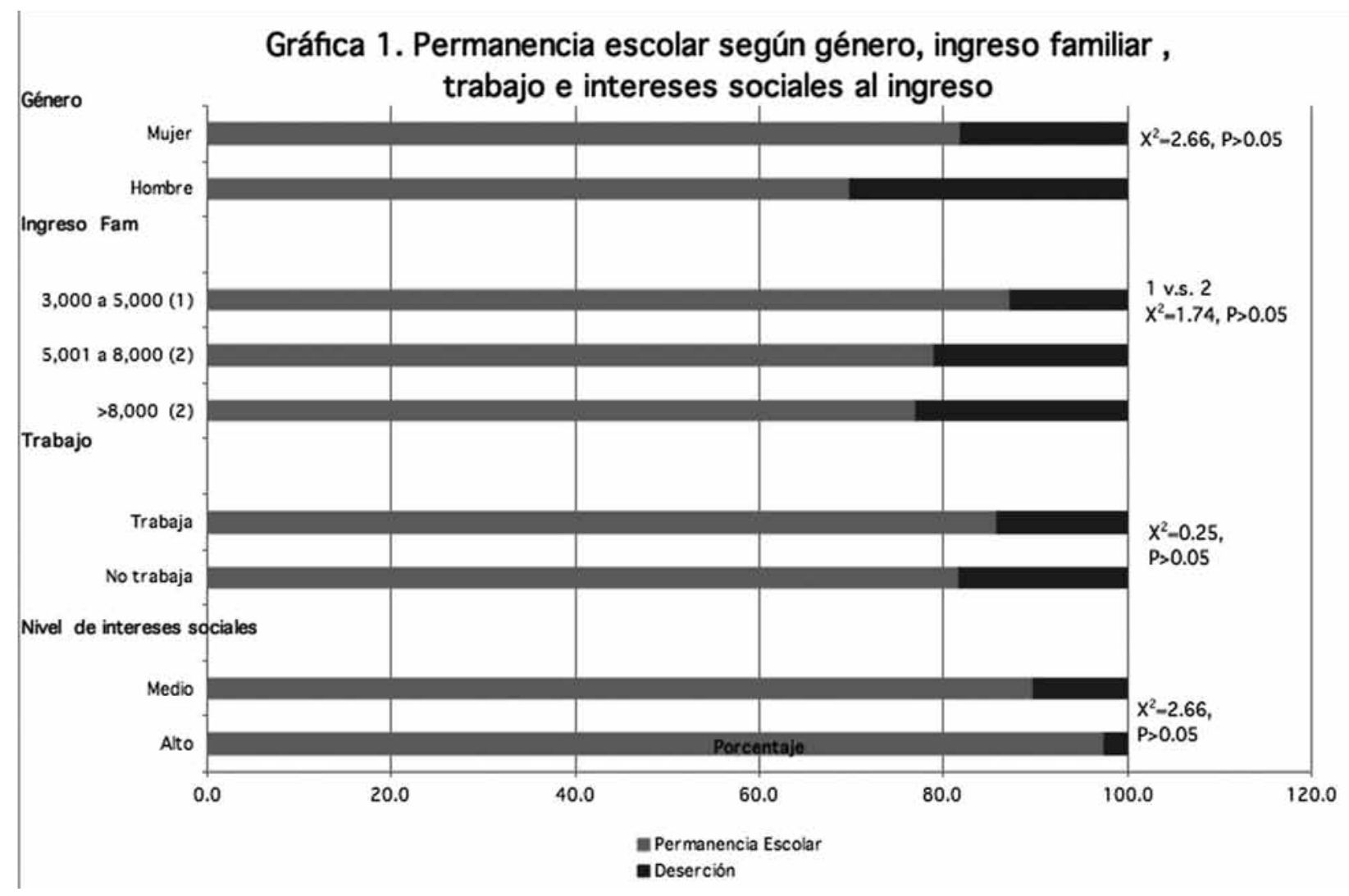




\section{Discusıón}

La profesión de Enfermería se ha caracterizado por el número insuficiente de profesionales en éste campo de la salud; lo que fundamenta la importancia de indagar sobre las características que poseen aquellas personas que aspiran formarse como licenciados en enfermería, dado que estos atributos personales impactan en su estadía escolar, eficiencia terminal y, en conjunto influyen en la deficiencia de personal en ésta rama, en México y en otros países.

El perfil observado en el grupo considerado (promedio de edad, estado civil, género y participación en el mercado laboral) tiene semejanza con el estudio de Ríos, Olivo y Moreno, ${ }^{10}$ es decir, el conjunto de estas características constituyen aspectos favorables para la estabilidad escolar y la permanencia en la carrera, debido a que permiten a los alumnos dedicarse a sus estudios; hay congruencia con la percepción de que enfermería es apropiada para la mujer. Así mismo, la procedencia de preparatorias públicas, es semejante con lo reportado por Marín, ${ }^{7}$ lo que presupone una población en riesgo de deserción debido a limitaciones en su desempeño escolar, ya que de acuerdo con la literatura revisada pareciera que los egresados de instituciones privadas tienen un mejor aprovechamiento, la evidencia proviene de un análisis sobre estudiantes que ingresaban a estudiar medicina, las mejores calificaciones en la prueba diagnóstica de conocimientos, pertenecieron a alumnos provenientes de escuelas privadas. ${ }^{13}$

En lo familiar y socioeconómico, hubo similitud con Ríos y Cols en la dependencia de los estudian-

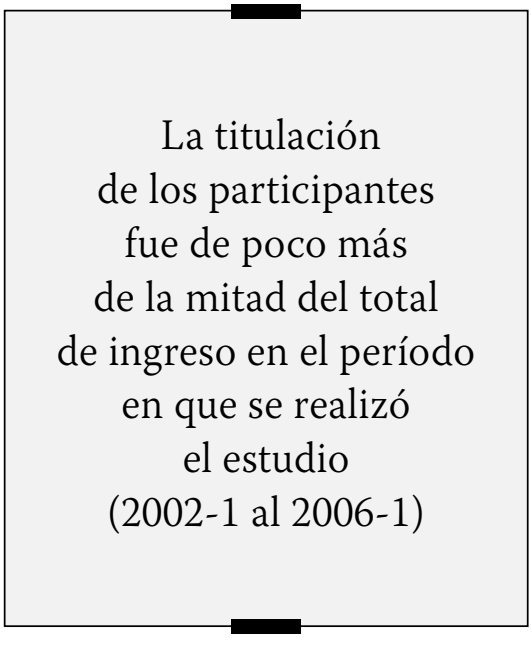

tes con sus padres, esto podría ser restrictivo en etapas más avanzadas de la vida pero por el contrario en la edad encontrada y siendo universitarios representa un gran soporte para alcanzar la formación profesional, sobretodo, al apoyarlos sus padres económicamente. Sobre la escolaridad de ambos padres, los resultados fueron inversos a los de Ríos y Col. en donde, la mayoría de los progenitores contaba con educación básica, mientras que en éste trabajo solo una minoría la obtuvo. Ramírez y Col. coinciden en la ocupación de los padres, como empleados, pero difieren en el nivel profesionista con una cifra menor a nuestro estudio. Por otra parte, conforme al ingreso familiar la mayoría de los hogares de estos estudiantes tienen condiciones económicas precarias (el más bajo salario mensual), y estos resultados son similares a lo encontrado por otros autores, ${ }^{8,10}$ como ya se ha señalado las condiciones económicas precarias pueden limitar el éxito escolar en algunos casos, aunque en otras ocasiones puede constituirse en un estímulo. No obstante, un aspecto que contrarresta estas condiciones es que, la mayoría de las familias cuentan con casa pro- pia y son familias pequeñas, lo que puede ser, hasta cierto punto, relajante para los escolares; coadyuvando así en una mayor productividad educativa.

El que el total de los jóvenes al ingresar a la carrera de LE manifestaron orientación hacia objetivos de conocimiento, seguido de lo social, hace similar los resultados a los reportados por Marín en cuanto a la necesidad de éxito intelectual que tenían los alumnos, continuado de lo social y biológico.

La titulación de los participantes fue de poco más de la mitad del total de ingreso en el período en que se realizó el estudio (2002-1 al 2006-1). De los cuales se titularon más mujeres que hombres. No obstante, en dicho periodo la eficiencia terminal y la deserción disminuyeron, esta última en mayor medida, de tal forma que la cifra es más cercana al promedio obtenido por la ANUIES. ${ }^{3}$ La remisión en la eficiencia terminal pudiera deberse a que es a partir del 2002 en el que se acordó llevar a cabo una titulación especial a través del programa del EGEL para abatir el número de egresados de años anteriores y colaborar a culminar así su formación profesional; de tal manera que se titularon y están en trámites de titulación con dicho programa, casi la cuarta parte de la población estudiada.

Si bien las diferencias observadas al analizar la permanencia escolar no alcanzaron significancia estadística, es posible considerarlas como una tendencia respecto a una mayor deserción de los varones y de quienes tienen mejores condiciones socioeconómicas. Quizá la situación económica que se percibe en la mayoría de los es- 
tudiantes que no desertaron constituye un reto y les brinda motivación, disposición y capacidad para concluir la carrera, como lo describe la teoría psicológica de Tinto; y no solamente diferencian los beneficios que obtendrán de acuerdo a los estudios universitarios, como lo señala la teoría económica. ${ }^{6}$

Se requiere evaluar la influencia de otros factores, por ejemplo en las estudiantes mujeres se ha observado que el embarazo, el matrimonio y con ello muchas veces la necesidad de trabajar para la manutención de su hijo pueden limitar u obstaculizar la permanencia escolar.

En síntesis a partir de estos datos podemos decir que nuestras suposiciones iniciales no se confirmaron, planteábamos que posiblemente carencias económicas o falta de apoyo familiar limitarían la permanencia escolar y lo observado ( $\sin$ que se obtuvieran diferencias estadísticamente significativas) es que desertaron en mayor medida los alumnos provenientes de hogares con mayores ingresos, en el grupo estudiado es predominante el residir y contar con el apoyo económico de los padres. La evaluación de los intereses y objetivos profesionales tampoco mostró datos concluyentes, habría que considerar la posibilidad de ampliar o mejorar el instrumento utilizado.

A pesar de que desertaron en menor porcentaje los alumnos con condiciones socioeconómicas más precarias, las instituciones escolares debieran apoyarlos en la medida de lo posible por lo que se sugiere que maestros tutores y autoridades institucionales brinden orientación sobre los diversos tipos de beca a las que puedan aspirar los jóvenes, para coadyuvar en el término de su educación profesional y con ello colaborar en la disminución de la deserción y eficiencia terminal.

\section{Referencias Bibliográficas}

1 Sanabria H. Deserción en estudiantes de enfermería en cuatro universidades de Perú. Anales de la Facultad de Medicina. 2002. [Consultado 13 mayo 2010 on line] 63 (4): [301-311]. Disponible en: http:// bit.ly/yWgffc

2 Nigenda G, Ruiz JA, Rosales Y,) Bejarano R. Enfermeras con licenciatura en México: estimación de los niveles de deserción escolar y desperdicio laboral. Salud Pública Méx 2006; Vol. 48(1):22-29

3 Rodríguez LJ, Hernández VJM. La deserción escolar universitaria en México. La experiencia de la Universidad Autónoma Metropolitana Campus Iztapalapa. Actualidades Investigativas en educación 2008. Revista Electrónica. [on line]. Abril. [Consultado 10 junio 2010]; 8 (1): [1-30]. Disponible en: http:// bit.ly/yZKzB9

4. Carbajal M, González LM, Hernández LA, Morales DE, Strempler L. Cambios de carrera en la UAM-X. Universidad Autónoma Metropolitana 2005. [Consultado 13 julio 2010] Disponible en: http://bit.ly/ MfX8ey

5 Verderese O, García J. La decisión de estudiar enfermería. 1971-1972. OPS-OMS. [Consultado 5 diciembre 2010] Disponible en: http://bit. ly/MBql0i

6 Romo LA, Fresán OM. Los factores curriculares y académicos relacionados con el abandono y el rezago. México: Asociación Nacional de Universidades e Instituciones de Educación Superior. Colección.
Biblioteca de la educación superior. Serie investigaciones. 2001. P: 244.

7 Marín JG. Perfil de nuevo ingreso como herramienta útil para el diseño de un programa institucional de Tutoría. [on line]. 2006 (Consultado 5 mayo 2011). Disponible en: http://bit.ly/zKpA01

8 Ramírez SE, Aguirre MG, Padilla LM, García De Alba JE. Diagnóstico situacional del estudiante de enfermería de la Universidad de Guadalajara. Rev Enferm Inst Mex Seguro Soc . 1992; 5 (1) 29-32.

9 Jara P, Polanco O, Alveal M. Percepción del rol profesional de alumnos de enfermería de la Universidad de Concepción de Chile. Invest Educ Enferm. 2005; 23 (2): 56-69.

10 Ríos B, Olivo JR, Romero SL. Los estudiantes de las unidades académicas de enfermería y medicina de la Universidad Autónoma de Nayarit. Un primer análisis a su perfil socioeconómico, cultural y laboral. 2010. Primer congreso Latinoamericano de Ciencias de la ducación. [on line] (Consultado 13 enero 2011]. Disponible en: http://bit.ly/ xgAVXE

11 Latiesa M. Demanda de educación superior: Evaluaciones y condicionamientos de los estudiantes en la elección de carrera. Revista Española de Investigaciones Sociológicas 1989; (46):1-39.

12Sigmarket. El ingreso en México. [on line]. 2008. [Consultado 13 enero 2010] Disponible en: http://bit.ly/ ctn0GQ

13 Gatica F, Méndez I, Sánchez M, Martínez A. Variables Asociadas al éxito académico en estudiantes de la licenciatura en Medicina de la UNAM. Revista de la facultad de Medicina de la UNAM. 2010; 53 (5): $9-18$ 